\title{
Contenidos obsesivos, miedo a la enfermedad y asco
}

\author{
Sandra Arnáez, Gemma García-Soriano y Amparo Belloch \\ Universidad de Valencia, Valencia, España
}

\begin{abstract}
Resumen: Los estudios recientes sobre la relación entre el trastorno obsesivo-compulsivo (TOC) y la emoción de asco han revelado algunas inconsistencias, que podrían deberse al papel diferencial que tienen la propensión y la sensibilidad al asco, a la influencia de terceras variables, o a la heterogeneidad del TOC. Este trabajo analiza la relación entre propensión y sensibilidad al asco, miedo a la enfermedad, y diferentes contenidos TOC en estudiantes universitarios $(N=114)$. La propensión al asco resulta ser la variable más importante en la predicción de los contenidos de Dudas/comprobación (19\% varianza explicada, VE), y el miedo a la enfermedad y la sensibilidad al asco lo son respecto a los contenidos de Limpieza/contaminación ( $21 \%$ y $8 \%$ VE, respectivamente) y Superstición (21\% y 24\% VE, respectivamente). Los resultados indican la necesidad de considerar las dimensiones del asco y el miedo a la enfermedad en la conceptualización, evaluación y tratamiento del TOC.
\end{abstract}

Palabras clave: TOC; propensión al asco; sensibilidad al asco; miedo a la contaminación; miedo a la enfermedad.

Obsessional contents, fear of illness and disgust

\begin{abstract}
Recently, data about the associations between disgust and obsessive-compulsive disorder (OCD) have show some inconsistencies, which might be due to the differential role of disgust sensitivity and propensity, the influence of other variables, or the heterogeneity of OCD contents. This study examines the relationships among disgust sensitivity and propensity, fear of illness, and different obsessional contents in university students $(N=114)$. Disgust propensity was the most relevant variable in predicting Doubt/checking contents (19\% of explained variance, EV), and fear of illness and disgust sensitivity were the most relevant variables predicting Contamination (EV: $21 \%$ and 8\%, respectively), and Superstitious contents (EV: $21 \%$ and $24 \%$, respectively). Results suggest the need to consider the disgust and fear of illness dimensions in the conceptualization, assessment and treatment programs of OCD.
\end{abstract}

Keywords: OCD; disgust propensity; disgust sensitivity; contamination fear; illness fear.

\section{Introducción}

El trastorno obsesivo-compulsivo (TOC) se caracteriza por la ocurrencia repetida de obsesiones y/ o compulsiones que causan gran malestar y ocupan el tiempo suficiente como para interferir significativamente en el

Recibido: 2 octubre 2014; aceptado: 11 diciembre 2014.

Correspondencia: Gemma García-Soriano, Dpto. Personalidad, Evaluación y Tratamiento Psicológicos, Facultad de Psicología, Universidad de Valencia, Av. Blaco Ibáñez 21, 47010 Valencia, España.

Correo-e: gemma.garcia@uv.es.

Agradecimientos: Este trabajo forma parte de los proyectos de investigación PSI2013-44733-R financiado por el Ministerio de Economía y Competitividad (Gobierno de España), y PROMETEO-2013/066, financiado por la Consejería de Educación, Cultura y Deporte (Generalitat Valenciana, Valencia, España). funcionamiento diario del individuo (DSM-5; American Psychological Association [APA], 2013). Tanto desde un punto de vista teórico (p.ej., Davey, 2011; McKay, 2006; Rachman, 2004) como empírico (p.ej., Olatunji, 2009; Tolin, Woods y Abramowitz, 2006), se ha sugerido que el TOC, o algunas formas del mismo, están asociadas con la emoción de asco en mayor medida que con el miedo y la ansiedad (Mancini, Gragnani y D'Olimpio, 2001). Esta asociación podría estar relacionada con la función adaptativa del asco como protector de la salud, al motivar la evitación de estímulos o situaciones potencialmente contagiosas o contaminantes (Sandín, Chorot, Santed, Valiente, y Olmedo, 2008b; Sandín et al., 2013a,b).

La mayor parte de las investigaciones que han estudiado el papel del asco en la sintomatología obsesi- 
vo-compulsiva (OC) han considerado esta emoción como un constructo unidimensiona ${ }^{1}$. Estos trabajos han encontrado, en población no clínica, que el asco se asocia de manera significativa con los síntomas de lavado y comprobación (por ej., Mancini et al., 2001; Tolin et al., 2006) y orden (Tolin et al., 2006), y que las personas que puntúan más en síntomas OC de contaminación presentan a su vez mayor puntuación en las medidas de asco (Olatunji, Lohr, Sawchuck y Tolin 2007). En población clínica, se ha constatado también que los pacientes con TOC y síntomas predominantes de contaminación muestran mayores niveles de asco que los pacientes TOC sin esos síntomas (Woody y Tolin, 2002). Otros trabajos han analizado la asociación entre asco y TOC teniendo en cuenta los estímulos desencadenantes del asco (por ej., aspectos relacionados con la higiene). En este sentido, se ha observado que la respuesta antes desencadenantes asociados a la higiene predice parte de la varianza de los síntomas OC de contaminación/ lavado y comprobación, y la dimensión moral las obsesiones de daño (Sandín et al., 2014).

No obstante, otros autores sugieren la necesidad de distinguir entre dos dimensiones de asco sin tener en cuenta los estímulos desencadenantes: la propensión al asco, definida como la frecuencia con la que se experimentan las sensaciones de asco, y la sensibilidad al asco, entendida como el malestar o miedo que produce la experiencia de asco (Van Overveld, de Jong, Peters, Cavanagh, y Davey, 2006). Ambas dimensiones se han asociado a la presencia de síntomas OC, aunque no hay acuerdo en la literatura respecto a si estas dimensiones se relacionan con todos los contenidos de síntomas OC, ni en si una de las dimensiones tiene un papel más relevante en alguno de los contenidos o dimensiones de síntomas OC. En este sentido,

${ }^{1}$ El término empleado por los autores que trabajan desde esta aproximación unidimensional es el de sensibilidad al asco, definida como la predisposición general a experimentar reacciones de asco o repugnancia, y evaluada especialmente a través de la Disgust Scale (DS; Haidt, McCauley y Rozin, 1994). Sin embargo, a lo largo de este trabajo, emplearemos el término genérico asco para referirnos a esta variable, que algunos autores han definido como más cercana al concepto de propensión que al de sensibilidad (van Overveld, de Jong y Peters, 2010; van Overveld, de Jong, Peters, Cavanagh y Davey, 2006). El término sensibilidad al asco se empleará exclusivamente referido a la dimensión descrita por quienes distinguen entre sensibilidad y propensión (frecuencia con la que se experimentan las sensaciones de asco) al asco, y que ha sido definida como el grado en que una persona experimenta como molesta la experiencia de sentir asco (Van Overveld et al., 2006). Frecuentemente ambas dimensiones, sensibilidad y propensión, se han evaluado a través de la Disgust Propensity and Sensitivity Scale-Revised (DPSS-R, van Overveld et al., 2006). algunos trabajos enfatizan la relación entre la propensión al asco y los síntomas OC de contaminación (Goetz et al., 2013; Moretz y McKay, 2008; Sandín, Chorot, Olmedo y Valiente, 2008a) y las puntuaciones elevadas en la escala de obsesiones del Obsessive-Compulsive Inventory-Revised (OCI-R; Foa et al., 2002) (Nicholson y Holmes, 2012). Además constatan que los pacientes con TOC presentan una mayor propensión al asco que otros pacientes ansiosos y que la población general (Olatunji, Tart, Ciesielski, McGrath y Smith, 2011). Sin embargo, otros estudios encuentran que es la sensibilidad al asco (y no la propensión), la dimensión que predice la presencia de síntomas OC de contaminación (p.ej., David et al., 2009; Nicholson y Holmes, 2012; Olatunji, 2010; Olatunji, Cisler, Deacon, Connolly y Lohr, 2007; Olatunji, Moretz, Wolitzky-Taylor, McKay, McGrath, Ciesielski, 2010) y obsesiones de daño (Sandín et al., 2008a). En suma, aunque estos resultados sugieren que posiblemente ambas dimensiones, propensión y sensibilidad, se relacionan de forma diferencial con los síntomas del TOC, y especialmente con los de contaminación, dada la disparidad de datos se precisan más estudios para poder establecer conclusiones válidas.

Por otro lado, también se ha postulado que los síntomas OC y en especial, los de contaminación, podrían tener alguna asociación con la ansiedad por la salud. Goetz et al. (2012) sugieren que los altos niveles de ansiedad por la salud potencian la relación entre los síntomas de contaminación y el asco al aumentar la preocupación sobre la propia integridad física que se experimenta con el miedo a la contaminación. Asimismo, Thorpe et al. (2011) proponen que el asco media en la asociación entre los síntomas OC y el miedo a la enfermedad. Estas propuestas difieren de la defendida por otros autores, que han sugerido que, en algunos pacientes TOC, los síntomas de contaminación se asocian a miedo a la enfermedad y derivan en reacciones ansiedad, mientras que en otros los síntomas de contaminación no se asocian con ese miedo a la enfermedad sino con la emoción de asco (Cougle, Wolitzky-Taylor, Lee, y Telch, 2007).

Por tanto, pese al relativamente elevado número de investigaciones que examinan la relación entre los síntomas OC y la experiencia de asco, quedan muchos interrogantes abiertos en torno a la naturaleza y el alcance de esa asociación. Especialmente, sigue sin resolverse si las dos dimensiones que se postulan para el asco se asocian de manera diferencial con las diferentes dimensiones de síntomas OC, así como el papel que juega en todo ello la ansiedad por la salud, una de las variantes del constructo clásico de hipocondría que ocupa un lugar es- 
pecial en la nueva nosología psiquiátrica que plantea el DSM-5 (APA, 2013).

El objetivo de este estudio es analizar la relación entre los diferentes contenidos o dimensiones de síntomas OC, las dimensiones de asco, y la ansiedad por la salud, específicamente el componente de miedo a la enfermedad. Para ello nos centraremos en los pensamientos intrusos de contenido obsesivo que experimenta la población general como análogos válidos de las obsesiones clínicas y de las preocupaciones hipocondríacas (Abramowitz, Fabricant, Taylor, Deacon, McKay, y Storch, 2014; García-Soriano, Belloch, y Morillo, 2008). Este enfoque nos permitirá examinar la naturaleza de las relaciones entre las variables de interés, minimizando los sesgos inherentes a realizar un estudio de esta naturaleza en población clínica (i.e., comorbilidad, respuesta a tratamientos, etc.).

\section{Método}

\section{Participantes}

La muestra estuvo formada por 114 personas que estaban cursando o habían finalizado estudios universitarios, con una media de edad de 27.47 años $(D T=11.39)$. La mayoría eran mujeres $(71 \%, n=81)$, permanecían solteros $(75 \%)$, y describieron su nivel socioeconómico como medio $(73 \%)$. No se incluyó a personas que manifestaron haber padecido o padecer algún trastorno mental y/ o estar siguiendo algún tipo de tratamiento psicofarmacológico o psicológico.

\section{Instrumentos}

La Escala de Propensión y Sensibilidad al Asco [Disgust Propensity and Sensitivity Scale-Revised, DPSS-R] (van Overveld et al., 2006) es un autoinforme de 16 ítems que evalúa la frecuencia y el impacto emocional de las experiencias de asco sin hacer alusión a estímulos desencadenantes específicos. La DPSS-R está compuesta por 2 subescalas que evalúan respectivamente propensión y sensibilidad al asco. La adaptación española del instrumento (Sandín et al., 2008a) ha mostrado una buena consistencia interna en el presente estudio (propensión al asco: $\alpha=0.83$; sensibilidad al asco: $\alpha=0.80$ ).

El Inventario de Pensamientos Intrusos Obsesivos (INPIOS; García-Soriano, 2008; García-Soriano, Belloch, Morillo y Clark, 2011) es un cuestionario de auto-informe que evalúa pensamientos, imágenes o impulsos intrusivos e indeseados que experimentan tanto personas de la población general como las personas con diagnóstico de TOC. El instrumento consta de dos par- tes. La primera está compuesta por 48 ítems que evalúan la frecuencia de aparición de diferentes pensamientos intrusos obsesivos (PIO) que se agrupan en seis factores dependiendo de su contenido: Agresivos; Religiosos, sexuales repugnantes, existenciales, y conductas socialmente inadecuadas; Orden/ precisión; Duda/ comprobación; Limpieza/ contaminación; y Superstición/ pensamiento mágico. En la segunda parte, los participantes valoran su intrusión más molesta e indican la frecuencia con la que emplean un listado de estrategias para controlarla (García-Soriano y Belloch, 2013). En el presente estudio únicamente se ha empleado la primera parte del instrumento, que ha mostrado una excelente consistencia interna con valores entre 0.83 (escala de PIO Superstición) y 0.90 (escala de PIO Religiosos/ sexuales).

El Índice de Sensibilidad a la Ansiedad-3 [Anxiety Sensitivity Index-3, ASI-3] (Taylor et al., 2007) consta de 18 ítems que, a través de 3 subescalas (física, cognitiva y social), evalúan las reacciones de miedo/ ansiedad ante la experiencia de síntomas de ansiedad de tipo físico, cognitivo y síntomas observables socialmente. En este estudio se ha empleado la puntuación total media con el objetivo de facilitar la interpretación de los resultados (Wheaton et al., 2012). La versión española (Sandín, Valiente, Chorot y Santed, 2007) ha mostrado una buena consistencia interna en el presente trabajo (puntuación total: $\alpha=0.86$ ).

El Inventario de Preocupaciones Hipocondríacas de Aiglé (IPHA) (Belloch, Fernández-Álvarez, y Castañeiras, 2004) es un autoinforme diseñado para evaluar las preocupaciones y miedos hipocondríacos en población general. Consta de 30 ítems agrupados en cinco escalas (miedo a la enfermedad/muerte, consciencia de hipocondría, atención/ vigilancia por la salud, vulnerabilidad a la enfermedad, y desconfianza hacia el médico y convicción de enfermedad). En el presente trabajo, únicamente se ha empleado la escala de miedo a la enfermedad/ muerte que evalúa miedos relacionados con la salud-enfermedad, cuya consistencia interna ha resultado satisfactoria $(\alpha=0.88)$.

\section{Procedimiento}

Para reclutar la muestra se ofreció participar en una investigación a 3 grupos de estudiantes de $3^{\circ}$ de Grado de Psicología. Los alumnos interesados completaron un protocolo de instrumentos que incluía, entre otros, los siguientes cuestionarios de autoinforme: DPSS-R, INPIOS ( $1^{\text {a }}$ parte $)$, ASI-3 e IPHA, junto a una hoja de datos personales y el consentimiento informado para participar en la investigación. El protocolo y procedi- 
miento fueron aprobados por la Comisión de Ética en Investigación Experimental de la Universidad.

\section{Resultados}

Asociaciones entre la frecuencia de los pensamientos intrusos obsesivos, las dimensiones de asco y el miedo a la enfermedad/ muerte

En primer lugar se analizaron las asociaciones entre la sensibilidad a la ansiedad y la frecuencia de las diferentes dimensiones de PIO (Tabla 1). Los resultados indicaron que la sensibilidad a la ansiedad (ASI-3) mantenía asociaciones moderadas con la frecuencia de los PIO de Duda/ comprobación, Limpieza/ contaminación y Superstición, y bajas pero significativas con la frecuencia de los PIO Agresivos. En segundo lugar se analizaron las asociaciones entre la frecuencia de los PIO y el resto de variables de interés (Tabla 1). Estos análisis se repitieron controlando el posible efecto de la sensibilidad a la ansiedad (Tabla 1) debido a la asociación significativa entre ASI-3 y algunas dimensiones de PIO, y a que se ha planteado que la sensibilidad a la ansiedad también podría constituir un factor de vulnerabilidad a desarrollar síntomas OC (p.ej., Deacon y Abramowitz, 2006). Los resultados indicaron relaciones moderadas entre las dos dimensiones de asco (propensión y sensibilidad) y la frecuencia de los PIO de Duda/ comprobación y de Limpieza/ contaminación, que se mantuvieron significativas una vez controlado el posible efecto de la sensibilidad a la ansiedad. Asimismo, se observaron asociaciones moderadas y significativas entre la sensibilidad al asco y los PIO-Superstición, que disminuyeron al controlar los efectos de la sensibilidad a la ansiedad.

A continuación exploramos si los PIO de Duda/ comprobación y Limpieza/ contaminación mantenían mayo- res asociaciones con alguna de las dos dimensiones de asco. Los resultados indican que la magnitud de las asociaciones entre sensibilidad y propensión al asco y la frecuencia de los PIO de Duda/ comprobación $(z=.955$, $p>.05)$ y Limpieza/ contaminación $(z=.120, p>.05)$ fueron similares.

Respecto a las asociaciones entre el miedo a la enfermedad/ muerte (IPHA) y la frecuencia de los diferentes contenidos obsesivos (INPIOS), se observaron asociaciones moderadas con la frecuencia de los PIO Limpieza/ contaminación y de Superstición, y bajas pero significativas con la frecuencia de los PIO Duda/ comprobación. Las asociaciones con los PIO Limpieza/ contaminación y de Superstición se mantuvieron significativas cuando se controló el posible efecto de la sensibilidad a la ansiedad.

\section{Predicción de la frecuencia de pensamientos intrusos} obsesivos a partir de las dimensiones de asco y el miedo a la enfermedad/ muerte

Con el fin de examinar qué dimensiones de síntomas OC eran predichos por el miedo a la enfermedad y las dos dimensiones de asco llevamos a cabo una serie de análisis de regresión múltiple empleando como variables dependientes las diferentes dimensiones de síntomas OC (INPIOS), e introduciendo como variables independientes la puntuación total del ASI-3, las escalas de la DPSS-R, y la escala de miedo a la enfermedad/ muerte del IPHA (Tabla 2). Dado el número de predictores y el tamaño de la muestra disponible, se empleó el procedimiento estadístico de pasos sucesivos.

Los resultados indicaron que la sensibilidad a la ansiedad únicamente predijo de forma significativa la frecuencia de los síntomas OC de agresión, explicando un $4 \%$ de la varianza $(F(1,111)=4.416, p<.038)$. Por

Tabla 1. Coeficientes de correlación (entre paréntesis las correlaciones parciales controlando ASI-3) entre las subescalas del INPIOS, las dimensiones de asco (DPSS-R) y la escala de miedo a la enfermedad/muerte (IPHA) $(N=114)$

\begin{tabular}{lcccc}
\hline \multirow{2}{*}{\multicolumn{1}{c}{ INPIOS }} & ASI-3 PTT & \multicolumn{2}{c}{ DPSS $-\mathrm{R}$} & $\begin{array}{c}\text { IPHA Miedo a la enfermedad/ } \\
\text { muerte }\end{array}$ \\
\cline { 3 - 4 } & & Propensión al asco & Sensibilidad al al asco & $.153(.04)$ \\
PIO Agresivos & $.196^{*}$ & $-.028(-.10)$ & $.179(.08)$ & $.119(.08)$ \\
PIO Sexuales, Religiosos & .096 & $-.073(-.11)$ & $.045(-.01)$ & $.141(.10)$ \\
PIO Orden/ Precisión & .114 & $.128(.10)$ & $.151(.10)$ & $.214^{*}(.05)$ \\
PIO Duda/Comprobación & $.297^{* *}$ & $.439^{* *}\left(.38^{* * *}\right)$ & $.330^{* *}\left(.26^{* *}\right)$ & $.457^{* * *}\left(.26^{* *}\right)$ \\
PIO Limpieza/ Contaminación & $.442^{* * *}$ & $.359^{* * *}\left(.25^{* *}\right)$ & $.373^{* *}\left(.25^{* *}\right)$ & $.457^{* * *}\left(.34^{* * *}\right)$ \\
PIO Superstición & $.347^{* * *}$ & $.139(.03)$ & $.343^{* *}(.18)$ & $.18)$ \\
\hline
\end{tabular}

Nota. PIO: Pensamientos Intrusos Obsesivos; INPIOS: Inventario de pensamientos intrusos obsesivos; DPSS-R: Escala de propensión y sensibilidad al asco-revisada; ASI-3: Índice de Sensibilidad a la Ansiedad-3 PT (puntuación total); IPHA: Inventario de Preocupaciones Hipocondríacas de Aiglé. $* p \leq .05 ; * * p \leq .01 ; * * * p \leq .001$. 
Tabla 2. Estadísticos de la ecuación final de regresión del INPIOS como variable dependiente y las dimensiones de asco (DPSS-R),

la sensibilidad a la ansiedad (ASI-3) y la escala de miedo a la enfermedad/muerte (IPHA) como variable independiente $(N=114)$

\begin{tabular}{lllllc}
\hline \multicolumn{1}{c}{ Variable Dependiente } & \multicolumn{1}{c}{ Variable Independiente } & $\Delta R^{2}$ & & $T$ & $p$ \\
\hline PIO Agresivos & ASI-3 PT & .038 & .196 & 2.101 & .038 \\
PIO Duda/ Comprobación & DPSS-R Propensión al asco & .193 & .439 & 5.146 & .000 \\
PIO Limpieza/ Contaminación & IPHA Miedo a la enfermedad/ muerte & .206 & .333 & 3.810 & .001 \\
& DPSS-R Sensibilidad al asco & .082 & .310 & 3.550 & .001 \\
PIO Superstición & IPHA Miedo a la enfermedad/ muerte & .212 & .386 & 4.282 & .001 \\
& DPSS-R Sensibilidad al asco & .244 & .193 & 2.138 & .035 \\
\hline
\end{tabular}

Nota. PIO: Pensamientos Intrusos Obsesivos; INPIOS: Inventario de pensamientos intrusos obsesivos; DPSS-R: Escala de propensión y sensibilidad al asco-revisada; ASI-3: Índice de sensibilidad a la ansiedad -3 PT (puntuación total); IPHA: Inventario de preocupaciones hipocondríacas de Aiglé.

otro lado, la propensión al asco explicaba un $19 \%(F(1$, $111)=26.480, p<.001)$ de la frecuencia con la que se experimentaban los PIO de Duda/ comprobación, mientras que la sensibilidad al asco explicaba un $8 \%$ de los PIO de Limpieza/ contaminación $(F(1,110)=22.219, p$ $<.001)$, y un $24 \%$ de los PIO de Superstición $(F(1,110)$ $=17.719, p<.001)$. Finalmente, el miedo a la enferme$\mathrm{dad} /$ muerte explicaba, respectivamente, un $20 \%$ y $21 \%$ de la frecuencia con la que se experimentan los PIO de Limpieza/ contaminación $(F(1,111)=28.818, p<.001)$ y Superstición $(F(1,111)=29.907, p<.001)$. Ninguna de las variables independientes predijo la frecuencia de los PIO de Simetría/ orden ni de los Sexuales/ religiosos.

\section{Discusión}

Diversos estudios han sugerido que tanto el asco (p. ej., Mancini et al., 2001; Olatunji, 2009) como el miedo a la enfermedad (p.ej., Davey y Bond, 2006; Thorpe et al., 2011) son variables relevantes en la aparición y/ o mantenimiento de los síntomas obsesivo-compulsivos, aunque no hay acuerdo sobre si estas variables son igualmente relevantes en todas las dimensiones de síntomas obsesivo-compulsivos. Es por ello que el objetivo del presente trabajo ha sido examinar la implicación de la propensión y la sensibilidad al asco y el miedo a la enfermedad en la frecuencia con la que se experimentan los pensamientos intrusos obsesivos análogos a los experimentados los pacientes con TOC, teniendo en cuenta los diferentes contenidos.

Hemos observado que la propensión y la sensibilidad al asco se asocian con la frecuencia de los PIO de Limpieza/ contaminación, Duda/ comprobación y Superstición. Estos resultados son consistentes con los informados previamente respecto a la contaminación (por ej., Goetz et al., 2013; Sandín et al., 2008a; Sandín et al., 2014). Además constatamos que el miedo a la enferme$\mathrm{dad} /$ muerte se relaciona con la frecuencia de los PIO de
Limpieza/ contaminación y de Superstición. Estos resultados son también consistentes con lo señalado por otros autores respecto a los contenidos de contaminación (Thorpe et al., 2003, 2011). Estas asociaciones se mantienen incluso una vez controlado el papel que juega la sensibilidad a la ansiedad, que ha sido señalada como otra variable de vulnerabilidad relevante en el TOC (p.ej., Deacon y Abramowitz, 2006; Taylor et al., 2007; Wheaton, Mahaffey, Timpano, Berman y Abramowitz, 2012). Cabe mencionar que la mayor parte de los estudios previos se han centrado en la relación entre estas variables y los síntomas de contaminación, no informando de la relación entre estas dimensiones y los síntomas de duda/ comprobación, y no analizando la asociación con los contenidos de superstición.

Los resultados obtenidos en los diferentes análisis de regresión confirman que tanto las dimensiones de asco como el miedo a la enfermedad/ muerte son variables relevantes únicamente en relación con la frecuencia con que se experimentan algunos contenidos obsesivos. Además sugieren que la sensibilidad a la ansiedad no tiene un papel demasiado relevante en la predicción de los PIO, explicando únicamente una pequeña parte (menor al 5\%) de la varianza de los de contenido agresivo. La dimensión de propensión al asco juega un papel más relevante, pero únicamente en la predicción de los PIO de Duda/ comprobación, y no en los de contaminación tal y como había sido sugerido por algunos estudios (por ej., Goetz et al., 2013; Moretz y McKay, 2008; Sandín et al., 2008a). El papel de la propensión al asco en la predicción de los síntomas de comprobación no ha sido hallada en trabajos previos empleando la DPSS-R, tal vez debido al empleo de instrumentos diferentes para evaluar la sintomatología de comprobación (por ej., Sandín et al., 2008a). Sin embargo, las relaciones entre el asco y los síntomas de comprobación sí han sido informadas previamente empleando la Disgust Scale (Mancini et al., 2001; Tolin et al., 2006). 
Por otro lado, la sensibilidad al asco y el miedo a la enfermedad predicen la frecuencia de los PIO de Limpieza/ contaminación y de Superstición. Estos resultados irían en la línea de los trabajos que han informado de un papel más relevante de la sensibilidad al asco (frente a la propensión) en la predicción de los síntomas OC de contaminación (por ej., Mitte, 2008; Olatunji, 2010; Olatunji, Cisler, Deacon, Connolly y Lohr, 2007), y también de los que han enfatizado la relevancia del miedo a la enfermedad en la expresión de los síntomas de contaminación (Cougle et al., 2007; Thorpe et al., 2011). De hecho, en este estudio, el miedo a la enfermedad/ muerte es la variable que predice un mayor porcentaje de la varianza de la frecuencia de los PIO de Limpieza/ Contaminación. Finalmente, cabe destacar que los modelos finales de regresión se ajustaron en mayor medida a la predicción de los PIO de Superstición que del resto de contenidos obsesivos, explicando en el primer caso un $46 \%$ de la varianza frente al $29 \%$ de los PIO de Limpieza/ contaminación. Este resultado es especialmente relevante debido a que la Superstición es a menudo la dimensión "olvidada" en los estudios sobre síntomas obsesivo-compulsivos (Summerfeldt, Kloosterman, Antony, Swinson, y Richter, 2004), y hasta donde nosotras sabemos, este es el primer estudio que analiza la relación entre asco, miedo a la enfermedad y PIO de Superstición.

A modo de conclusión, cabe señalar que los resultados de nuestro trabajo ponen de relevancia el papel diferencial que la propensión y la sensibilidad al asco y el miedo a la enfermedad desempeñan en la predicción de algunos contenidos de pensamientos intrusos obsesivos: Duda/ comprobación, Limpieza/ contaminación y Superstición. Estos resultados tendremos que tomarlos con precaución dado el empleo de una muestra no clínica, lo que dificulta la generalización de los resultados obtenidos a población clínica, si bien es cierto que autores relevantes en este ámbito apoyan y defienden el uso de muestras no clínicas para la investigación tanto del TOC (p.ej., Abramowitz et al., 2014; Gibbs, 1996) como de la relación entre los síntomas OC y el asco (por ej., Olatunji, 2010; Wheaton et al., 2012), especialmente cuando los constructos que se evalúan tienen naturaleza dimensional, como es el caso de este trabajo. Además, cabe destacar que los instrumentos empleados en este estudio (i.e., DPSS-R, INPIOS, IPHA) asumiendo la dimensionalidad de las variables evaluadas, han sido desarrollados y validados para ser empleados tanto en población clínica como no clínica. No obstante, asumimos la necesidad de ampliar y reproducir estudios como el que se presenta aquí utilizando población clínica, de manera que sea posible realizar generalizaciones acerca de nuestras conclusiones.
Los resultados de este estudio indican también la necesidad de tener en consideración las dimensiones de asco y del miedo a la enfermedad a la hora de evaluar la sintomatología obsesiva, y desarrollar programas de tratamiento para el TOC. Asimismo, sugieren la necesidad de tener en cuenta el contenido de las obsesiones (y compulsiones) predominantes en los pacientes con TOC a la hora de desarrollar tratamientos específicos, sin olvidar que, incluso dentro de un mismo subgrupo, pueden existir diferencias en la presentación de los síntomas, siendo necesarios enfoques de tratamiento diferentes en dichos casos (Sookman, Abramowitz, Calamari, Wilhelm, y McKay, 2005).

\section{Referencias}

Abramowitz, J. S., Fabricant, L. E., Taylor, S., Deacon, B. J., McKay, D., y Storch, E. A. (2014). The utility of analogue studies for understanding obsessions and compulsions. Clinical Psychology Review, 34(3), 206-217. doi:10.1016/j. cpr.2014.01.004

Abramowitz, J. S., y Deacon, B. J. (2006). Psychometric properties and construct validity of the obsessive-compulsive inventory--revised: Replication and extension with a clinical sample. Journal of Anxiety Disorders, 20(8), 1016-1035. doi:http://dx.doi.org/10.1016/j.janxdis.2006.03.001

American Psychiatric Association (2013). Diagnostic and Statistical Manual of Mental Disorders. 5 th edition. Washington, DC: American Psychiatric Association.

Ato, M., López, J. J., \& Benavente, A. (2013). Un sistema de clasificación de los diseños de investigación en psicología. Anales de Psicología, 29(3), 1038-1059.

Belloch, A., Fernández-Álvarez, H., y Castañeiras, C (2004). Presentación de un instrumento para la detección y valoración de las preocupaciones hipocondríacas en la población general: El inventario de preocupaciones hipocondríacas Revista Argentina de Clínica Psicológica, 13(3), 183-195.

Berle, D., y Phillips, E. S. (2006). Disgust and obsessive-compulsive disorder: An update. Psychiatry: Interpersonal and Biological Processes, 69(3), 228-238. doi:http://dx.doi. org/10.1521/psyc.2006.69.3.228

Cougle, J. R., Wolitzky-Taylor, K., Lee, H., y Telch, M. J. (2007). Mechanisms of change in ERP treatment of compulsive hand washing: Does primary threat make a difference? Behaviour Research and Therapy, 45(7), 1449-1459. doi:http://dx.doi. org/10.1016/j.brat.2006.12.001

Davey, G. C. (2011). Disgust: the disease-avoidance emotion and its dysfunctions. Philosophical Transactions of the Royal Society B: Biological Sciences, 366(1583), 3453-3465.

Davey, G. C. L., y Bond, N. (2006). Using controlled comparisons in disgust psychopathology research: The case of disgust, hypochondriasis and health anxiety. Journal of Behavior Therapy and Experimental Psychiatry, 37(1), 4-15. doi:http:// dx.doi.org/10.1016/j.jbtep.2005.09.001

David, B., Olatunji, B. O., Armstrong, T., Ciesielski, B. G., Bondy, C. L., y Broman-Fulks, J. (2009). Incremental specifi- 
city of disgust sensitivity in the prediction of obsessive-compulsive disorder symptoms: Cross-sectional and prospective approaches. Journal of Behavior Therapy and Experimental Psychiatry, 40(4), 533-543. doi:http://dx.doi.org/10.1016/j. jbtep.2009.07.004

Foa, E. B., Huppert, J. D., Leiberg, S., Langner, R., Kichic, R., Hajcak, G., y Salkovskis, P. M. (2002). The Obsessive-Compulsive Inventory: development and validation of a short version. Psychological assessment, 14(4), 485.

García-Soriano, G. (2008) Obsessional intrusive thoughts inventory (INPIOS). American Psychological Association: PsycTESTS.

García-Soriano, G., y Belloch, A. (2013). Symptom dimensions in obsessive-compulsive disorder: differences in distress, interference, appraisals and neutralizing strategies. Journal of Behavior Therapy and Experimental Psychiatry, 44(4), 441448.

García-Soriano, G., Belloch, A., y Morillo, C. (2008). Sobre la heterogeneidad del trastorno obsesivo-compulsivo: una revisión. Revista de Psicopatología y Psicología Clínica, 13(2), 65-84.

García-Soriano, G., Belloch, A., Morillo, C., y Clark, D. A. (2011). Symptom dimensions in obsessive-compulsive disorder: From normal cognitive intrusions to clinical obsessions. Journal of Anxiety Disorders, 25(4), 474-482. doi:http://dx. doi.org/10.1016/j.janxdis.2010.11.012

Gibbs, N. A. (1996). Nonclinical populations in research on obsessive-compulsive disorder: A critical review. Clinical Psychology Review, 16(8), 729-773.

Goetz, A. R., Lee, H. J., Cougle, J. R., y Turkel, J. E. (2013). Disgust propensity and sensitivity: Differential relationships with obsessive-compulsive symptoms and behavioral approach task performance. Journal of Obsessive-Compulsive and Related Disorders, 2(4), 412-419. doi:10.1016/j.jocrd.2013.07.006

Mancini, F., Gragnani, A., y D’Olimpio, F. (2001). The connection between disgust and obsessions and compulsions in a non-clinical sample. Personality and Individual Differences, 31(7), 1173-1180. doi:http://dx.doi.org/10.1016/S01918869(00)00215-4

McKay, D. (2005). Treating disgust reactions in contamination-based obsessive-compulsive disorder. Journal of Behavior Therapy and Experimental Psychiatry, 37(1), 53-59. doi:http:// dx.doi.org/10.1016/j.jbtep.2005.09.005

Mitte, K. (2008). Trait-disgust vs. fear of contamination and the judgmental bias of contamination concerns. Journal of Behavior Therapy and Experimental Psychiatry, 39(4), 577-586. doi: http://dx.doi.org/10.1016/j.jbtep.2008.01.005

Nicholson, E., y Barnes-Holmes, D. (2012). Developing an implicit measure of disgust propensity and disgust sensitivity: Examining the role of implicit disgust propensity and sensitivity in obsessive-compulsive tendencies. Journal of Behavior Therapy and Experimental Psychiatry, 43(3), 922-930. doi:http:// dx.doi.org/10.1016/j.jbtep.2012.02.001

Olatunji, B. O. (2009). Incremental specificity of disgust propensity and sensitivity in the prediction of health anxiety dimensions. Journal of Behavior Therapy and Experimental Psychiatry, 40(2), 230-239. doi:http://dx.doi.org/10.1016/j. jbtep. 2008.10 .003
Olatunji, B. O. (2010). Changes in disgust correspond with changes in symptoms of contamination-based OCD: A prospective examination of specificity. Journal of Anxiety Disorders, 24(3), 313-317. doi:http://dx.doi.org/10.1016/j.janxdis.2010.01.003

Olatunji, B. O., Cisler, J. M., Deacon, B. J., Connolly, K., y Lohr, J. M. (2007). The disgust propensity and sensitivity scale-revised: Psychometric properties and specificity in relation to anxiety disorder symptoms. Journal of Anxiety Disorders, 21(7), 918-930. doi:http://dx.doi.org/10.1016/j.janxdis.2006.12.005

Olatunji, B. O., Lohr, J. M., Sawchuk, C. N., y Tolin, D. F. (2007). Multimodal assessment of disgust in contamination-related obsessive-compulsive disorder. Behaviour Research and Therapy, 45(2), 263-276. doi:http://dx.doi.org/10.1016/j. brat.2006.03.004

Olatunji, B. O., Tart, C. D., Ciesielski, B. G., McGrath, P. B., y Smits, J. A. J. (2011). Specificity of disgust vulnerability in the distinction and treatment of OCD. Journal of Psychiatric Research, 45(9), 1236-1242. doi:http://dx.doi.org/10.1016/j. jpsychires.2011.01.018

Rachman, S. (2004). Fear of contamination. Behaviour Research and Therapy, 42(11), 1227-1255.

Sandín, B., Chorot, P., Olmedo, M., y Valiente, R. M. (2008a). Escala de propensión y sensibilidad al asco-revisada (DPSS-R): propiedades psicométricas y relación del asco con los miedos y los síntomas obsesivo-compulsivos. Análisis y modificación de conducta, 34, 127-168.

Sandín, B., Chorot, P., Santed, M. A., Valiente, R. M., y Olmedo, M. (2008b). Sensibilidad al asco: Concepto y relación con los miedos y los trastornos de ansiedad. Revista de Psicopatología y Psicología Clínica, 13(3), 137-158. doi: 10.5944/rppc. vol.13.num.3.2008.4056.

Sandín, B., Chorot, P., Valiente, R. M., \& Santed, M. A. (2014). Dimensiones de sensibilidad al asco y predicción diferencial de los síntomas del trastorno obsesivo-compulsivo. Ansiedad y Estrés, 20(1), 11-25.

Sandín, B., Chorot, P., Valiente, R., Santed, M., Olmedo, M., Pineda, D., y Campagne, D. (2013a). Construcción y validación preliminar de la escala multidimensional de sensibilidad al asco (EMA). Revista de Psicopatología y Psicología Clínica, 18(1), 1-18. doi: 10.5944/rppc.vol.18.num.1.2013.12759.

Sandín, B., Valiente, R. M., Chorot, P., y Santed, M. A. (2007). ASI-3: Nueva escala para la evaluación de la sensibilidad a la ansiedad. Revista de Psicopatología y Psicología Clínica, 12(2), 91-104. doi: 10.5944/rppc.vol.12.num.2.2007.4036.

Sandín, B., Valiente, R. M., Santed, M. A., Pineda, D., \& Olmedo, M. (2013b). Efecto de la sensibilidad al asco sobre los síntomas hipocondríacos: un estudio prospectivo. Cuadernos de Medicina Psicosomática y Psiquiatría de Enlace, 107, 32-45.

Sookman, D., Abramowitz, J. S., Calamari, J. E., Wilhelm, S., y McKay, D. (2005). Subtypes of obsessive-compulsive disorder: Implications for specialized cognitive behavior therapy. Behavior Therapy, 36(4), 393-400. doi:http://dx.doi. org/10.1016/S0005-7894(05)80121-2

Summerfeldt, L. J., Kloosterman, P. H., Antony, M. M., Swinson, R. P., y Richter, M. A. (2004). The relationship between miscellaneous symptoms and major symptom factors in obsessive-compulsive disorder. Behavior Research and Therapy, 42, 1453-1476. 
Thorpe, S. J., Barnett, J., Friend, K., y Nottingham, K. (2011). The mediating roles of disgust sensitivity and danger expectancy in relation to hand washing behaviour. Behavioural and Cognitive Psychotherapy, 39(2), 175-190. doi:http://dx.doi. org/10.1017/S1352465810000676

Thorpe, S. J., Patel, S. P., y Simonds, L. M. (2003). The relationship between disgust sensitivity, anxiety and obsessions. Behaviour Research and Therapy, 41(12), 1397-1409. doi:http:// dx.doi.org/10.1016/S0005-7967(03)00058-5

Tolin, D. F., Woods, C. M., y Abramowitz, J. S. (2006). Disgust sensitivity and obsessive-compulsive symptoms in a non-clinical sample. Journal of Behavior Therapy and Experimental Psychiatry, 37(1), 30-40. doi:http://dx.doi.org/10.1016/j.jbtep.2005.09.003

Van Overveld, M., de Jong, P. J., Peters, M. L., y Schouten, E. (2011). The Disgust Scale-R: A valid and reliable index to in- vestigate separate disgust domains?. Personality and Individual Differences, 51(3), 325-330.

Van Overveld, W. J. M., de Jong, P. J., Peters, M. L., Cavanagh, K., y Davey, G. C. L. (2006). Disgust propensity and disgust sensitivity: Separate constructs that are differentially related to specific fears. Personality and Individual Differences, 41(7), 1241-1252. doi:http://dx.doi.org/10.1016/j.paid.2006.04.021

Wheaton, M. G., Mahaffey, B., Timpano, K. R., Berman, N. C., y Abramowitz, J. S. (2012). The relationship between anxiety sensitivity and obsessive-compulsive symptom dimensions. Journal of Behavior Therapy and Experimental Psychiatry, 43(3), 891-896. doi:http://dx.doi.org/10.1016/j.jbtep.2012.01.001

Woody, S. R., y Tolin, D. F. (2002). The relationship between disgust sensitivity and avoidant behavior: Studies of clinical and nonclinical samples. Journal of Anxiety Disorders, 16(5), 543559. doi:http://dx.doi.org/10.1016/S0887-6185(02)00173-1 